\title{
ON SEMIGROUPS IN ANALYSIS AND GEOMETRY
}

\section{CHARLES LOEWNER ${ }^{1}$}

Introduction. The investigations which I wish to report are concerned with certain transformation semigroups, particularly with extensions of some classical groups to semigroups. The concept "transformation semigroup" will be used in the following sense: let $V$ be a manifold and let $\subseteq$ be a set of local homeomorphisms in $V$, i.e., homeomorphisms between two domains (open connected sets) in $V$. We call $\subseteq$ a semigroup if the following conditions (a) to (d) are satisfied.

(a) If $f \in \subseteq$ maps $O_{1}$ onto $O_{2}$ and $g \in \subseteq$ maps the domain $O_{2}$ onto $\mathrm{O}_{3}$, then the composite mapping $g \circ f$, which transforms $O_{1}$ onto $O_{3}$, belongs to $\mathbb{S}$ also.

(b) If $O_{1}$ is any subdomain of the domain $O$ of $f \in \mathfrak{S}$, then $f$ restricted to $\mathrm{O}_{2}$ belongs to $\widetilde{S}$ also.

(c) The identity map of $V$ belongs to $\mathfrak{S}$.

(d) If a sequence of mappings $f_{n} \in \mathfrak{S}$, all defined in the same domain $O$, converges uniformly in any compact part of $O$ and the limit $f$ is again a homeomorphism of $O$, then $f$ belongs to $\subseteq$ also.

It would conform better to the usual terminology to call $\subseteq$ a pseudo-semigroup. For the sake of simplicity of language we drop the prefix "pseudo" and speak of a semigroup in $V$ instead. If all the maps of $\mathfrak{S}$ are restrictions of maps defined on the whole of $V$, we shall speak of a semigroup on $V$. In this case, only the maps on the whole of $V$ need to be considered.

We shall say that a semigroup $\subseteq$ in $V$ can be characterized locally if the further condition is satisfied.

(e) If the domain $O$ is the union $\mathrm{U}_{\alpha} O_{\alpha}$ of some domains $O_{\alpha}$, and a homeomorphism $f$ of $O$, restricted to any $O_{\alpha}$, belongs to $\mathfrak{S}$, then $f$ belongs to $\subseteq$ in the whole $O$.

If the inverse of any $f$ of a semigroup $\mathfrak{S}$ in $V$ also belongs to $\mathfrak{S}$, then we speak of a group in $V$. If, in particular, $\subseteq$ is a semigroup on $V$, the transformations in $\subseteq$ on the whole of $V$ map $V$ onto itself and form a topological group of $V$ in the ordinary sense.

An address delivered before the Vancouver meeting of the Society on August 30, 1962, by invitation of the Committee to Select Hour Speakers for Summer and Annual Meetings; received by the editors November 26, 1962 and, in revised form, August $10,1963$.

1 This work was supported in part by National Science Foundation Grant Number 14134 at Stanford University. 
We shall give some examples of the introduced concepts. Let $\mathfrak{B}$ be the set of all orientation-preserving projective transformations

$$
y=\frac{\alpha x+\beta}{\gamma x+\delta}(\alpha \delta-\beta \gamma>0)
$$

applied to intervals not containing the pole. The transformations represent a group in the Euclidean line $E_{1}$. An example of a semigroup, which will occur in later considerations, is given by the set of all orientation-preserving homeomorphisms between intervals in $E_{1}$ which do not decrease the cross ratio of any four naturally ordered points. As an example of a semigroup on the $n$-dimensional real vector space $R_{n}$ we mention the set of all nonsingular linear maps which, in a suitable coordinate system, are represented by matrices with nonnegative elements and a nonzero determinant. We may also characterize these linear maps as those which transform positive vectors (vectors with nonnegative components) into positive vectors. In general, we can associate with each convex cone ${ }^{2} C \subset R_{n}$ a semigroup $\mathfrak{S}_{c}$ consisting of those nonsingular linear maps which transform $C$ into itself. In the special case mentioned before, $C$ is the cone $P_{n}$ of positive vectors of $R_{n}$. Another example of a semigroup on $V=R_{n}$ is given by the set of all nonsingular linear maps which, in a suitable coordinate system, are represented by totally positive matrices, i.e., matrices whose minors are all nonnegative, and whose determinant is positive.

1. Extension of groups to minimal semigroups. After all the fundamental definitions have been introduced, we shall now start our discussions with the following question: we know from the Lie-group theory that the group $\mathfrak{B}$ in $E_{1}$, introduced before, has only one proper extension to a group of orientation-preserving homeomorphisms of intervals in $E_{1}$, namely the group of all orientation-preserving homeomorphisms. On the other hand, it is clear that there exist many proper extensions of $\mathfrak{P}$ to semigroups. The semigroup mentioned before, consisting of transformations not decreasing the cross ratio of naturally ordered quadruples of points, is an example of such an extension. It is now natural to ask for proper extensions of $\mathfrak{P}$ to semigroups $\mathfrak{S}$ which are minimal in the following sense: There is no proper extension of $\mathfrak{P}$ to a semigroup contained in $\mathfrak{S}$, but not coinciding with it. I determined the minimal extensions which satisfy the condition that they contain a map which is of class $C^{3}$ and does not belong to $\mathfrak{P}$. Then the following theorem holds [13]:

2 The use of the concept "cone" is explained in $\$ 1$. 
THEOREM I. There are only two such minimal extensions, ${ }^{8}$ say $\mathbb{S}^{\prime}$ and $\mathfrak{S}^{\prime \prime}$, one consisting of the inverses of the mappings of the other. $\mathfrak{S}^{\prime}$ consists of the (real) analytic mappings of intervals of $E_{1}$ which can be analytically continued into the upper half plane and map the latter schlicht into itself.

I conjecture that the theorem holds without any differentiability assumption.

We may ask whether it is possible to generalize the theorem to higher dimension in the following sense: let $\mathfrak{P}_{n}(n>1)$ be the group of orientation-preserving projective mappings of domains of the Euclidean space $E_{n}$ into $E_{n}$. What are its minimal extensions? The answer is disappointing. The only minimal extensions of $\mathfrak{P}_{n}$ are the group of all projectivities and the group of all orientation-preserving homeomorphisms of domains of $E_{n}$ into $E_{n}$. This again can easily be proved if only extensions are considered that contain a map not in $\mathfrak{B}_{n}$ which is of class $C^{3}$.

If one analyzes the proof of Theorem I, one realizes that the failure of the generalization to higher dimension is due to the following fact: the points in $E_{1}$ are naturally ordered, but not those in $E_{n}(n>1)$. In order to get interesting results in higher dimensions it is therefore necessary to add to the structure of $E_{n}$ an order relation which allows at least a partial ordering. The simplest and most important order relation results from the addition of a convex cone $C$ to the structure of $E_{n}$. A convex cone in $E_{n}$ is a set of vectors closed under linear combination with nonnegative coefficients. We shall further assume that the cone is closed in the natural topology and is after exclusion of the vertex completely contained in the interior of a half space whose boundary contains the origin. The order $x<y$ of two points $x$ and $y$ in $E_{n}$ with respect to $C$ is defined by the requirement that the vector $y-x$ lies in $C$. The order relation is evidently transitive, reflexive and asymmetric, but, for $n>1$, only a partial one since there are pairs of points $x$ and $y$ for which neither $x<y$ nor $y<x$ holds.

A mapping $f$ of a domain $O$ in $E_{n}$, in which an order relation with respect to a convex cone $C$ has been introduced, will be called $C$ monotonic if for any pair of points $x_{1}$ and $x_{2}$ in $O$ the relation $x_{1}<x_{2}$ implies that $f\left(x_{1}\right)<f\left(x_{2}\right)$.

We introduce further the concept of locally $C$-monotonic mappings in $E_{n}$ : a mapping defined in a domain $O$ of $E_{n}$ is locally $C$-monotonic if for any point of $O$ it is $C$-monotonic in a sufficiently small neighborhood of that point. A $C$-monotonic mapping of a domain is also locally

Containing a map of class $C^{3}$ and not belonging to $\mathfrak{B}$. 
monotonic, but the converse is not necessarily true, even if the mapping is $1-1 .^{4}$ This is true only if $n=1$ where the order is complete.

The $C$-monotonic homeomorphisms of domains in $E_{n}$ and also the locally $C$-monotonic homeomorphisms form semigroups. In the language given in the introduction, the latter semigroup can be characterized locally. Only semigroups of locally $C$-monotonic mappings will be considered in this and the following section.

First it is of interest to know the groups of locally $C$-monotonic homeomorphisms whose inverses are of the same type. The natural generalization of Theorem I would then ask for minimal extensions of such groups to locally $C$-monotonic semigroups. We shall discuss several special cases which have a natural place in classical analysis and geometry.

2. Semigroups connected with some special cones.

(A) We assume first that $C=L_{n}$ is a quadratic cone in $E_{n}(n>1)$, consisting of vector $\xi$ whose coordinates $\left(x^{1}, x^{2}, \cdots, x^{n}\right)$ in a suitable coordinate system satisfy the inequalities

$$
\left(x^{n}\right)^{2}-\sum_{j=1}^{n-1}\left(x^{j}\right)^{2} \geqq 0, \quad x^{n} \geqq 0 .
$$

In view of its connection with light propagation, $L_{n}$ is called the $n$ dimensional light cone. The order based on it is, in case $n=4$, identical with the time order of the special theory of relativity.

We introduce the group $B L_{n}$ of all homeomorphisms of domains in $E_{n}$ which with their inverses are locally $L_{n}$-monotonic. The cases $n=1$ and 2 play an exceptional role. We shall discuss them later. If $n>2$, the following assertion can be made: $\oiint_{L_{n}}(n>2)$ is a finite dimensional group of dimension $(n+1)(n+2) / 2$. Its transformation $\bar{x}^{i}=f_{i}\left(x^{1}, x^{2}, \cdots, x^{n}\right)(i=1,2, \cdots, n)$ can be characterized by two properties:

$(\alpha)$ The differential form $\left(d x^{n}\right)^{2}-\sum_{j=1}^{n-1}\left(d x^{j}\right)^{2}$ is invariant modulo a positive factor (in general not constant).

( $\beta) d x^{n} \geqq 0$ implies $d \bar{x}^{n} \geqq 0$.

The Lorentz transformations are the linear transformations of $\oiint_{L_{n}}$ keeping $(\alpha)$ absolutely invariant. One obtains the whole group $\mathcal{H}_{L_{n}}$ by forming composites of Lorentz transformations and the special transformation

$$
\bar{x}^{i}=-\frac{x^{i}}{\left(x^{n}\right)^{2}-\sum_{i=1}^{n-1}\left(x^{i}\right)^{2}} \quad(i=1,2, \cdots, n) .
$$

${ }^{4}$ Counterexamples can easily be constructed. 
REMARK. Formally one obtains the transformation of $G_{L_{n}}$ from the transformations of the $n$-dimensional Moebius group by replacing $x^{j}$ by $i x^{i}$ and $\bar{x}^{j}$ by $i \bar{x}^{i}(j=1,2, \cdots, n-1)$, where $i$ is the imaginary unit. But here we operate only in the real domain.

The natural problem corresponding to that solved for the group $\mathfrak{B}$ by Theorem I would be to find all minimal extensions of $B_{L_{n}}$ to locally $L_{n}$-monotonic semigroups.

Before we can describe solutions of this problem, we have to introduce a certain distinguished semigroup $\Xi_{C}$ associated with any convex cone $C$. It is a subsemigroup of the semigroup $\widetilde{S}_{C}$ defined in the introduction. Let $\alpha$ be any nonzero vector on the boundary of $C$. It may be multiplied by any positive factor without losing this property. Further let $\beta$ be a covariant vector representing a hyperplane of support of $C$ containing $\alpha$. We normalize $\beta$ up to a positive factor by requiring that the scalar product $(\beta, \xi)$ with any $\xi \in C$ be nonnegative. Now we form the Kronecker product $\alpha \otimes \beta$. It represents a mixed tensor of order 2, or a linear map associated with the pair of vectors $\alpha$ and $\beta$. If a coordinate system is used and $a^{i}$ are the components of $\alpha$ and $b_{j}$ the componens of $\beta$, then $\alpha \otimes \beta$ is represented by the matrix

$$
\left(c_{j}^{i}\right)=\left(a^{i} b_{j}\right) \quad(i, j=1, \cdots, n) .
$$

One sees immediately that the corresponding linear mappings $\bar{\xi}=\alpha(\xi, \beta)$, or written in coordinates $\bar{x}^{i}=a^{i} \sum_{j=1}^{n} b_{j} x^{j}(i=1,2, \cdots, n)$, map all vectors $\xi \in C$ into $\alpha$ multiplied by a nonnegative factor and, therefore, map $C$ into itself. We form now the convex hull $\Gamma_{C}$ of all $\alpha \otimes \beta$ constructed in the described way including the zero tensor. It represents a convex cone in the space of mixed tensors of order 2 or, alternately, in the space of linear homogeneous maps of $E_{n}$.

We associate now with $\Gamma_{C}$ a semigroup on $E_{n}$ in the following way: we consider the elements of $\Gamma_{C}$ as representing infinitesimal transformations of the group of linear homogeneous transformations and form all composites of them by solving differential equations

$$
\frac{d \xi}{d t}=\gamma(t) \xi \quad(0 \leqq t \leqq \alpha, \alpha>0)
$$

where $\gamma(t) \in \Gamma_{C}$ represents a one-parameter family of elements of $\Gamma_{C}$. We may assume that $\gamma(t)$ is $L_{1}$-integrable in $0 \leqq t \leqq \alpha$ and $\xi(t)$ absolutely continuous. The linear map giving $\xi(\alpha)$ of the solutions of (3) in terms of the initial value $\xi(0)$ is said to be generated by composition of the infinitesimal transformations $\gamma(t) .{ }^{5}$ By admitting all possible

s "Composition" and "integration" of infinitesimal transformations are used as synonyms. 
$\gamma(t)$ we add to the set of all transformations obtained in this way their limits in the natural topology. We recognize easily that the system of transformations thus closed represents a semigroup $\xi_{C}$ of linear transformations contained in $\boldsymbol{S}_{c}$. More than that, the transformations of $\xi_{C}$ map a vector $\xi \in C$ into a vector $\xi^{\prime}>\xi$. (In the language of projective geometry the semigroup $\varepsilon_{C}$ was introduced in [11].)

We are now able to give the solution of the posed problem under the additional condition: there exists an infinitesimal transformation of $\subseteq$ which is of class $C^{\prime}$ and is not an infinitesimal transformation of $\mathbb{B}_{I_{n}}$. The following theorem holds:

THEOREM II. There exists only one such minimal extension of $\$ I_{n}$ $(n \geqq 3)$ to a semigroup of locally $L_{n}$-monotonic transformations. It is the least semigroup containing both ${ }^{\$} L_{n}$ and $B_{L_{n}}$. We may also say that it consists of those transformations which can be composed of finitely many transformations taken from $\$_{L_{n}}$ and $\Sigma_{L_{n}}$ and their limits in the sense of uniform convergence in compact parts of the domain in which the transformations are defined.

The concept "infinitesimal transformation" for arbitrary semigroups $\gamma$ will always be used in the following sense: let $f_{t}: \bar{x}^{i}=f_{i}\left(x^{1}, x^{2}, \cdots, x^{n}, t\right)(0 \leqq t \leqq \alpha, \alpha>0)$ be a one-parameter family of transformations $f_{t}$ of $\subseteq$ of a fixed domain $O$ and $f_{0}$ the identity mapping. Assume further that the derivatives

$$
\left(\partial f_{i} / \partial t\right)_{t=0}=\xi^{i}\left(x^{1}, x^{2}, \cdots, x^{n}\right)
$$

exist. Then the vector field with components $\xi^{i}$ in $O$ represents an infinitesimal transformation of $\mathfrak{\subseteq}$.

It is probable that the assumption of the existence of the infinitesimal transformation of class $C^{\prime}$ can be replaced by the purely topological condition that there exists a mapping in $\mathbb{S}$ which is not in (5) $L_{n}$ and which is homotopic to the identity mapping in the class of mappings of its domain $O$ belonging to $\mathbb{S}$.

We have so far excluded the cases $n=1$ and $n=2$. If $n=2$, we may bring $L_{2}$ into the position of the first quadrant $x^{1} \geqq 0, x^{2} \geqq 0$ by rotating the coordinate system by the angle $\pi / 4$. Then it appears natural to consider $L_{2}$ and $L_{1}$ as special cases of the $n$-dimensional cone $P_{n}(n=1,2, \cdots)$ of positive vectors described by the inequalities $x^{1} \geqq 0, x^{2} \geqq 0, \cdots, x^{n} \geqq 0$, and to study the $P_{n}$-monotonic transformations. This will now be done.

(B) The group $\overbrace{P_{n}}$ consisting of all homeomorphisms of domains in $E_{n}$, which with their inverses are locally $P_{n}$-monotonic, is here 
infinite-dimensional. It consists of those transformations which in the neighborhood of a point can be described by equations $\bar{x}^{i}=\phi^{i}\left(x^{\mu_{i}}\right)(i=1,2, \cdots, n)$ where the $\phi^{i}$ are continuous and strictly increasing in some intervals and the $x^{\mu i}$ represent a permutation of the $x^{i}$.

Instead of operating with $\mathscr{S}_{P_{n}}$, we will introduce the finite-dimensional subgroup $\mathfrak{B}_{n}$ of $\mathbb{S}_{P_{n}}$ of transformations

$$
\bar{x}^{i}=l^{i}\left(x^{\mu_{2}}\right)=\frac{\alpha_{i} x^{\mu_{i}}+\beta_{i}}{\gamma_{i} x^{\mu_{i}}+\delta_{i}},\left|\begin{array}{cc}
\alpha_{i} & \beta_{i} \\
\gamma_{i} & \delta_{i}
\end{array}\right|>0 \quad(i=1,2, \cdots, n) .
$$

If $n=1$, the group $\mathfrak{P}_{1}$ coincides with the group $\mathfrak{P}$ with which we started our discussion. For any $n$ the group $\mathfrak{F}_{n}$ has the fundamental property that it cannot be properly extended to a finite-dimensional group of higher dimension.

We consider minimal extensions of $\mathfrak{P}_{n}$ to semigroups of locally $P_{n}$-monotonic transformations. The case $n=1$ is settled by Theorem I. Under assumption that there exists an infinitesimal transformation of $\subseteq$ of class $C^{3}$ which is not an infinitesimal transformation of $\mathfrak{B}_{n}$ $(n \geqq 2)$ the following theorem holds:

THEOREM III. For $n \geqq 2$ there exist three such minimal extensions of $\mathfrak{P}_{n}$ to a semigroup of locally $P_{n}$-monotonic transformations:

(a) the least semigroup containing $\mathfrak{P}_{n}$ and $\mathbb{B}_{P_{n}}$;

(b) the semigroup consisting of the transformations locally describable by

$$
\bar{x}^{i}=\phi^{i}\left(x^{\mu_{i}}\right) \quad(i=1,2, \cdots, n)
$$

where the $\phi^{i}$ are defined in some intervals $\left(a_{i}, b_{\imath}\right)$ and belong to the onedimensional semigroup $\mathfrak{S}^{\prime}$, which occurs in Theorem I, and the $\mu_{i}$ are a permutation of the indices;

(c) the semigroup consisting of the inverses of the mappings of (b). (We may also say that the $\phi_{i}$ belong to $\mathfrak{S}^{\prime \prime}$.)

We were led to problem (B) from problem (A) by considering the special cases $n=1$ and $n=2$. We shall now consider a new type of problems arising from the discussion of the cases where the dimensions are equal to 3 and 4 in problem (A). In view of the role of the cones (1) in these special cases in physics we shall call the coordinates $x, y, t$ or $x, y, z, t$, respectively, with $t$ being the time variable and $x, y, z$ the spatial coordinates. The inequalities (1) can then be expressed in the following simple way:

If $n=3$, the real symmetric matrix 


$$
\left(\begin{array}{cc}
t+x & y \\
y & t-x
\end{array}\right)>0
$$

If $n=4$, the Hermitian matrix

$$
\left(\begin{array}{ll}
t+x & y+i z \\
y-i z & t-x
\end{array}\right)>0
$$

The inequalities $\left(1^{\prime}\right)$ and $\left(1^{\prime \prime}\right)$ mean that the corresponding quadratic or Hermitian forms, respectively, are nonnegative. Indeed, the nonnegativity of the forms is completely expressed by the nonnegativity of their traces and their determinants, which expresses in each case the equivalent of (1). The time order of special relativity theory is thus identified with the order of quadratic or Hermitian forms in two variables, if the relation $A_{1}<A_{2}$ for two forms $A_{1}$ and $A_{2}$ is defined by the nonnegativity of the difference form $A_{2}-A_{1}$. It is now natural to generalize the considerations to the spaces of quadratic or Hermitian forms in any number of variables $n>1$ and base an order relation on them on the convex cone $Q_{n}$ of all negative quadratic forms or on the cone $H_{n}$ of all nonnegative Hermtiian forms, respectively. The (real) dimensions of the spaces are $N=n(n+1) / 2$ in the first case and $N=n^{2}$ in the second case.

We shall now consider the semigroups associated with them.

(C) We have first to describe the groups $\$_{Q_{n}}\left(\mathbb{S}_{H_{n}}\right)$ of all homeomorphisms of domains in $E_{N}$ which with their inverses are locally $Q_{n}\left(H_{n}\right)$-monotonic. It turns out that the groups are in both cases of finite dimension. We shall describe them by using coordinate systems. A quadratic form is then represented by a real symmetric matrix and a Hermitian form by a Hermitian matrix.

(a) The group ${ }^{B} Q_{n}$ is composed of linear homogeneous transformations $A \rightarrow B$ of the form

$$
B=C A C^{\prime}
$$

where $C$ is any real nonsingular matrix and $C^{\prime}$ its transpose, and the transformations

$$
B=\frac{\alpha A+\beta}{\gamma A+\delta} \quad(\alpha, \beta, \gamma, \delta \text { real and } \alpha \delta-\beta \gamma>0)
$$

applied to domains in which the denominator matrix $\gamma A+\delta$ is nonsingular.

The transformations $\left(6^{\prime}\right)$ simply represent transitions to new coordinate systems. Instead of the whole system $\left(6^{\prime \prime}\right)$ one may put in 
only the transformation $B=-A^{-1}$.

(b) The group $\$_{H_{n}}$ is composed of transformations of the following three types:

$$
B=C A C^{*}
$$

where $C$ is any nonsingular complex matrix, and $C^{*}=\bar{C}^{\prime}$ its conjugate transpose.

$\left(7^{\prime \prime}\right)$ The transformations $\left(6^{\prime \prime}\right)$, applied here to Hermitian matrices $A$.

( $\left.7^{\prime \prime \prime}\right) \quad$ The transition to the conjugate matrix $B=\bar{A}$.

Again it would be sufficient to put into $\left(7^{\prime \prime}\right)$ only the transformation $B=-A^{-1}$.

We again assume that only extensions of $\mathbb{S}_{Q_{n}}\left(\mathcal{S}_{H_{n}}\right)$ are considered which satisfy the following condition: there exists an infinitesimal transformation of class $C^{\prime}$, which is not an infinitesimal transformation of $S_{Q_{n}}\left(S_{H_{n}}\right)$. Then the following theorem holds:

THEOREM IV. There exists only one such minimal extension of $\left(\$ Q_{Q_{n}}\left(\$_{H_{n}}\right)\right.$ ( $\left.n \geqq 2\right)$ to a semigroup of locally $Q_{n}\left(H_{n}\right)$-monotonic transformations. It is the least semigroup containing both $\left(_{Q_{n}}\left(\mathcal{S}_{H_{n}}\right)\right.$ and $\varepsilon_{Q_{n}}\left(B_{H_{n}}\right)$.

3. Monotonic mappings of higher order in $E_{1}$. Among the transformations in the space of quadratic or Hermitian forms, a particularly important role is played by those which can be associated with scalar functions: let $f(t)$ be a real valued function of a real variable $t$, varying in an interval $(a, b)$. Let further $A$ be a quadratic (Hermitian) form in $n$ dimensions with eigenvectors $e_{i}(i=1,2, \cdots, n)$ and corresponding eigenvalues $\lambda_{i}$ which all fall into the interval $(a, b)$. Then $f(A)$ is defined as the quadratic (Hermitian) form with the same eigenvectors $e_{i}$ and eigenvalues $f\left(\lambda_{i}\right)$. This definition leads to a transformation $X \rightarrow f(X)$ whose domain is the totality of quadratic (Hermitian) forms of dimension $n$ whose eigenvalues fall into $(a, b)$. We call $f(t)$ monotonic of order $n$ in $(a, b)$ if the function $f(X)$ is $Q_{n}\left(H_{n}\right)$ monotonic, [8] or [12]. It turns out that this concept does not depend on whether we use quadratic or Hermitian forms. The class of monotonic functions of order $n$ can be completely characterized by the following difference inequalities: a function $f(t)$ is monotonic of order $n$ in $(a, b)$ if for any values

$$
a<x_{1}<y_{1}<x_{2}<y_{2}<\cdots<x_{m}<y_{m}<b \quad(m \leqq n)
$$

the determinant 


$$
\left|\frac{f\left(x_{i}\right)-f\left(y_{j}\right)}{x_{i}-y_{j}}\right|_{i, j=1}^{m} \geqq 0 .
$$

Monotonicity of order $n$ implies monotonicity of any lower order. Monotonic functions of order 1 are simply the nondecreasing functions in the ordinary sense. One can derive from (8) that monotonic functions of order $n>1$ are of class $C^{2 n-3}$ and that, after exclusion of constant functions, they have a strictly positive first derivative. We shall call the class of mappings represented by them $\mathfrak{M}_{n}$. By $\mathfrak{M}_{1}$ we shall mean the class of mappings represented by continuous strictly increasing functions. From the definition of monotonicity of order $n$ it follows immediately that $\mathfrak{M}_{n}$ is a semigroup in $E_{n}$. It is further clear that

$$
\mathfrak{M}_{1} \supset \mathfrak{M}_{2} \supset \mathfrak{M}_{3} \supset \cdots
$$

and that the intersection $\mathfrak{M}_{\infty}$ of all $\mathfrak{M}_{n}(n=1,2, \cdots)$ is also a semigroup. One can also easily show that the semigroup thus obtained is no more restricted, if one operates in Hilbert space and uses the usual definition of $f(A)$ for a selfadjoint operator $A$ with spectrum in $(a, b)$. The special case of a fractional power was considered by E. Heinz [20] and T. Kato [21]. $\mathfrak{M}_{\infty}$ is of particular interest because of the following

Theorem V. $A$ map $f(t)$ defined in $(a, b)$ belongs to $\mathfrak{M}_{\infty}$ if and only if it is analytic in $(a, b)$ and from there can be analytically continued into the full upper half plane, and maps the latter into itself.

An elementary proof of Theorem $\mathrm{V}$ is contained in [8]. A proof based on theorems on unbounded operators in Hilbert space was given by A. Koranyi and B. Sz.-Nagy, [4] and [5]. A connection of the theorem with Hamburger's moment problem is discussed by $\mathrm{J}$. Bendat and S. Sherman in [1].

We observe that the semigroup $\mathfrak{S}^{\prime}$, which appears in Theorem I, is a proper subsemigroup of $\mathfrak{M}_{\infty}$. It consists of those mappings of $\mathfrak{M}_{\infty}$ whose analytic continuations into the upper half plane map the latter schlicht into itself. It is of interest to ask whether the restriction of $\mathfrak{M}_{\infty}$ to $\mathfrak{S}^{\prime}$ can be described without leaving the real domain. This is indeed the case, as is expressed by the following theorem [13]:

TheOREM VI. The maps of $\mathfrak{S}^{\prime}$ are thase maps of $\mathfrak{M}_{\infty}$ which can be obtained by composition of infinitesimal transformations of $\mathfrak{M}_{\infty}$.

$\mathfrak{S}^{\prime}$ is a proper subsemigroup of $\mathfrak{M}_{\infty}$. From this fact one may expect that also $\mathfrak{M}_{n}$ for sufficiently high $n$ cannot be completely generated by composition of its infinitesimal transformations. The precise result is expressed in 
THEOREM VII. $\mathfrak{M}_{1}$ and $\mathfrak{M}_{2}$ can be completely generated by composition of its infinitesimal transformations. This is not true for any $\mathfrak{M}_{n}$ with $n>2$.

The maps of $\mathfrak{M}_{n}$, which can be generated by infinitesimal transformations of $\mathfrak{M}_{n}$, form a subsemigroup $\mathfrak{S}_{n} \subset \mathfrak{M}_{n}$. The Theorems I and $\mathrm{V}$ suggest calling the transformations of $\mathfrak{S}_{n}$ schlicht montonic of order $n$. Only for $n=1$ and $n=2$ do the concepts of monotonicity and schlicht monotonicity describe the same class of mappings.

4. Semigroups invariant under given groups. We shall now discuss some problems which branch off in a natural way from the introduction of the semigroup $\mathfrak{B}_{L_{n}}$ connected with the light cone $L_{n}(n \geqq 3)$. $\&_{L_{n}}$ is a semigroup of linear homogeneous transformations which transform $L_{n}$ into itself. We introduce further the group \&5 of homogeneous Lorentz transformations, which transform $L_{n}$ onto itself. It is clear that $\Sigma_{L_{n}}$ is invariant under (\$)

If we introduce the concept of the Lorentz norm of a vector $\xi=\left(x^{1}, x^{2}, \cdots, x^{n}\right)>0$ by the formula

$$
|\xi|=\left[\left(x^{n}\right)^{2}-\sum_{j=1}^{n-1}\left(x^{j}\right)^{2}\right]^{1 / 2}
$$

we may characterize the transformations of $(S)$ as those linear homogeneous transformations which keep the norm invariant and do not change the sign of $x^{n}$. They are completely determined by their action on the Lorentz unit sphere $S_{n-1}:|\xi|=1, x^{n}>0$, which is kept invariant. In this way we may interpret the transformations of $\$$ as the isometries of the $(n-1)$-dimensional hyperbolic non-Euclidean geometry with $S_{n-1}$ as the underlying space and the metric described by the Minkowskian line element

$$
d s=\left[\sum_{j=1}^{n-1}\left(d x^{j}\right)^{2}-\left(d x^{n}\right)^{2}\right]^{1 / 2}
$$

Essentially we deal with the Klein model of the hyperbolic nonEuclidean geometry in $(n-1)$ dimensions, in which the points of $S_{n-1}$ are replaced by the half rays emanating from the origin passing through them. The half rays are then interpreted as points of the $(n-1)$-dimensional projective space.

Now we transplant also the transformations of $L_{L_{n}}$ onto $S_{n-1}$ by observing how the intersections of half rays in $L_{n-1}$ with $S_{n-1}$ are transformed. In this way we obtain a semigroup of transformations on the non-Euclidean space $S_{n-1}$, invariant under its isometries. Comparing this with the Euclidean geometry, we may think of the follow- 
ing well-known fact: there are only two nontrivial invariant subgroups of the group of Euclidean isometries, the group of translations and the group of orientation-preserving isometries. Now it can be shown that not even a nontrivial invariant subsemigroup of the group of isometries of the hyperbolic geometry exists. We may, therefore, consider the semigroup $\varepsilon_{L_{n}}$, transplanted in the previously described way onto $S_{n-1}$, as a substitute for the translation group of the Euclidean geometry in the hyperbolic non-Euclidean geometry.

We now take a more abstract point of view and look, in general, for semigroups on $S_{n-1}$ which are invariant under the group of isometries. In particular, we are interested in such semigroups which share with the already constructed one certain simple properties. To understand them, we make the following preparatory remarks: the infinitesimal transformation of a Lie-group form a finite-dimensional linear space and its dimension is identical with that of the group. The infinitesimal transformations of a semigroup form a closed convex cone and its dimension may be finite even if the dimension of the semigroup is infinite. By the dimension of a convex cone we mean the dimension of its linear hull.

We now formulate the problem we wish to solve: find all closed convex cones $\Gamma$ of infinitesimal transformations in the space of the hyperbolic geometry which satisfy the conditions:

(a) The transformations generated by $\Gamma$ map the whole space into itself.

(b) $\Gamma$ is finite-dimensional.

(c) $\Gamma$ is invariant under the group of isometries.

(d) There is no nontrivial subcone of $\Gamma$ of lower dimension satisfying (c).

The problem was solved for the two-dimensional case in [13] and [14], and the solution may be described in the following way: we introduce in $E_{3}$ the Lorentz harmonics, i.e., the forms in $\xi\left(x^{1}, x^{2}, x^{3}\right)$ which satisfy the wave equation

$$
\frac{\partial^{2} \phi}{\partial\left(x^{3}\right)^{2}}-\frac{\partial^{2} \phi}{\partial\left(x^{1}\right)^{2}}-\frac{\partial^{2} \phi}{\partial\left(x^{2}\right)^{2}}=0 .
$$

The Lorentz harmonics of degree $n$ form a linear space $H_{n}$ of dimension $2 n+1 . H_{n}$ is the linear hull of the special harmonics $(\beta, \xi)^{n}$ $=\left(b_{1} x^{1}+b_{2} x^{2}+b_{3} x^{3}\right)^{n}$ formed with covariant vectors $\beta\left(b_{1}, b_{2}, b_{3}\right)$ representing planes of support of $L_{3}$, i.e., $b_{3}^{2}-b_{1}^{2}-b_{2}^{2}=0$. We will assume $b_{3}>0$. We form the convex hull $h_{n} \subset H_{n}$ of these special harmonics. We associate now with any $\phi \in h_{n}$ the Lorentz gradient 


$$
\nabla_{L}\left(\phi /|\xi|^{n}\right)
$$

i.e., the contravariant vector field obtained from the ordinary gradient by changing the signs of the first two components. By $r=|\xi|$ we mean, as before, the Lorentz norm of $\xi$. Since $\phi / r^{n}$ is homogeneous of order zero, $\nabla_{L}\left(\phi / r^{n}\right)$ is at points of $S_{2}$ tangential to $S_{2}$ and, therefore, may be considered as representing an infinitesimal transformation on $S_{2}$. Since $h_{n}$ is a convex cone of functions on $S_{2}$, the infinitesimal transformations thus constructed from all $\phi \in h_{n}$ form also a convex cone. Call it $\sigma_{n}$. We construct new vector fields from those of $\sigma_{n}$ by rotating each vector on $S_{2}$ in the sense of the Minkowskian metric either by $\pi / 2$ or $-\pi / 2$. The new vector fields form then a linear space $\tau_{n}$ of infinitesimal transformations on $S_{2}$. We now formulate the solution of our problem in

TheOREM VIII. The only closed convex cones of infinitesimal transformations on $S_{2}$, which satisfy the conditions (a), (b), (c) and (d) are the $\sigma_{n}$ and $\tau_{n}(n=1,2, \cdots)$. (See [14].)

One can easily identify the semigroup on $S_{\mathbf{2}}$ associated with $\varepsilon_{L_{3}}$ with the semigroup generated by $\sigma_{2}$. Its dimension is eight. The semigroup generated by $\sigma_{1}$ is six-dimensional. It is best described by changing from the Klein-model to the Poincaré-model of the two-dimensional hyperbolic geometry. Here the group of isometries appears as the group of Moebius transformations of the complex z-plane, which map the unit disc $|z|<1$ onto itself. The semigroup generated by $\sigma_{1}$ appears there as a semigroup of Moebius mappings of the unit disc into itself [13].

In his doctoral thesis [2] C. Ballantine determined all semigroups of Moebius transformations transforming $|z|<1$ into itself, which are invariant under the group of isometries and completely generated by their infinitesimal transformations. They depend on one continuous parameter.

The linear space $\tau_{1}$ of infinitesimal transformations generates the group of proper isometries. All other solutions of our problem generate semigroups or groups of infinite dimension. The $\sigma_{n}(n>2)$ generate semigroups proper (i.e., not groups). All $\tau_{n}(n>1)$ generate the same group, namely the group of all orientation and area-preserving transformations in the non-Euclidean plane. The area is taken in the sense of the non-Euclidean metric.

An example of a semigroup invariant under the group of isometries, which is completely generated by an invariant cone of infinitesimal transformations which is infinite-dimensional and which cannot be 
replaced by a smaller convex cone without diminishing the generated semigroup, is given by the conformal mappings of the unit circle $|z|<1$ onto schlicht domains lying in this circle [7].

5. Order relation connected with a given semigroup. The semigroups considered in $\$ \S 1$ and 2 were based on an a priori given order. On the other hand, we can derive in a natural way from each given transformation semigroup $\subseteq$ a partial order between points of the space $V$ on which $\subseteq$ operates. We shall assume here that $\subseteq$ is a semigroup on $V$. With a group $\subseteq$ on $V$ an equivalence relation is connected. Two points $p$ and $q$ in $V$ are equivalent if there exists a transformation of $\subseteq$ which transforms $p$ into $q$. The points equivalent to a given point $p$ form the orbit of $p$. If $\subseteq$ is only a semigroup on $V$, the condition that $p$ is transformable into $q$ by a transformation of $\mathfrak{S}$, this relation being designated by writing $p<q$, represents only a partial order. This order is of interest only if $\subseteq$ is not transitive, i.e., if $p<q$ is not always true. But if $\subseteq$ is transitive, we may go over to new semigroups by considering the action of $\subseteq$ on figures in $V$, such as system of a finite number of points in $V$, or if $V$ is a smooth manifold, the action on tensors or even more general geometrical entities. If the semigroup extended to the space of those figures is not transitive, new order relations arise. With any such order relation extremum problems are connected if we wish to determine the boundary of the set of successors of a given point $p$ in $V$ or in the space of the chosen figures. We shall give here some examples:

EXAMPLE 1. Restriction of a certain problem on parabolic partial differential equations to finite dimensions leads to the consideration of the semigroup in the $n$-dimensional real vector space $R_{n}$ generated by the infinitesimal transformations $A \xi$, where $A$ is any nonpositive self-adjoint operator vanishing on $\epsilon(1,1, \cdots, 1)$. The linear mappings generated form a semigroup of doubly stochastic operators. I determined in [15] the successors of $(1,0, \ldots, 0)$ in any dimension $n$ and, for $n=2$, J. Ryff constructed in his doctoral thesis [16] the successors of any $\xi$.

EXAMPLE 2. In [14] I considered the order relations connected with the semigroup generated by $\sigma_{1}$ (see $\$ 4$ ) in the hyperbolic nonEuclidean plane. The semigroup itself is transitive, but intransitive in its action on contravariant vectors. I determined the successors of a vector which are attached to the same point.

If a semigroup $\subseteq$ contains infinitesimal transformations, one may modify the associated order relations $p<q$ by requiring that the transition from $p$ to $q$ may be obtained by composition of infinitesimal transformations of $\mathfrak{\Xi}$. This requires the construction of the subsemi- 
group $\mathfrak{S}^{*}$ of $\mathfrak{S}$ of transformations which can be obtained by composition of infinitesimal transformations of $\mathfrak{\Im}$. This is in general a difficult problem. In this connection I would like to mention that the semigroup of totally positive operators can be completely generated by its infinitesimal transformations [18] and [10].

Final Remark. Theorems II, III, IV, V and VII are new. The proofs will be given in two other papers.

\section{REFERENCES}

1. J. Bendat and S. Sherman, Monotone and convex operators, Trans. Amer. Math. Soc. 79 (1955), 58-71.

2. C. Ballantine, Some Moebius semigroups on the 2-sphere, J. Math. Mech. 11 (1962), 455-467.

3. O. Dobsch, Matrixfunktionen beschränkter Schwankung, Math. Z. 43 (1937), 353-388.

4. A. Korányi, On a theorem of Löwner and its connections with resolvents of selfadjoint transformations, Acta Sci. Math. (Szeged) 17 (1956), 63-70.

5. B. Sz.-Nagy, Remarks to the preceding paper of A. Koranyi, Acta Sci. Math. (Szeged) 17 (1956), 71-75.

6. F. Kraus, Über konvexe Matrixfunktionen, Math. Z. 41 (1936), 18-42.

7. C. Loewner (=K. Löwner), Über schlichte konforme Abbildungen des Inheitskreises, Math. Ann. 89 (1923), 103-121.

8. - Über monotone Matrixfunktionen, Math. Z. 38 (1934), 177-216.

9. - Some classes of functions defined by difference or differential inequalities, Bull. Amer. Math. Soc. 56 (1950), 308-319.

10. - On totally positive matrices, Math. Z., 63 (1955), 338-340.

11. - On some transformation semigroups, J. Rational Mech. Anal. 5 (1956), 791-804.

12. - Advanced matrix theory, mimeographed notes, Stanford Univ., Stanford, Calif., 1957.

13. - Semigroups of conformal mappings, Seminars on analytic functions, Vol. 1, pp. 278-288, Institute for Advanced Study, Princeton, N. J., 1957.

14. - On some transformation semigroups invariant under Euclidean and nonEuclidean isometries, J. Math. Mech. 8 (1959), 393-409.

15. - A theorem on the partial order derived from a certain transformation semigroup, Math. Z. 72 (1959), 53-60.

16. J. V. Ryff, Semigroups generated by contracting vector fields, Tech. Rep. No. 1, Stanford Univ., Stanford, Calif., 1962.

17. - Ordinary differential equations determined by contracting vector fields, J. Math. Anal. Appl. 6 (1963), 43-54.

18. A. M. Whitney, A reduction theorem for totally positive matrices, J. Analyse Math. 2 (1952), 88-92.

19. E. P. Wigner and J. von Neumann, Significance of Loewner's theorem in the quantum theory of collisions, Ann. of Math. (2) 59 (1954), 418-433.

20. E. Heinz, Beiträge zur Storungstheorie der Spektralzerlegung, Math. Ann. 123 (1951), 415-438.

21. T. Kato, Notes on some inequalities for linear operators, Math. Ann. 125 (1952), 208-212.

STANFORD UNIVERSITY 